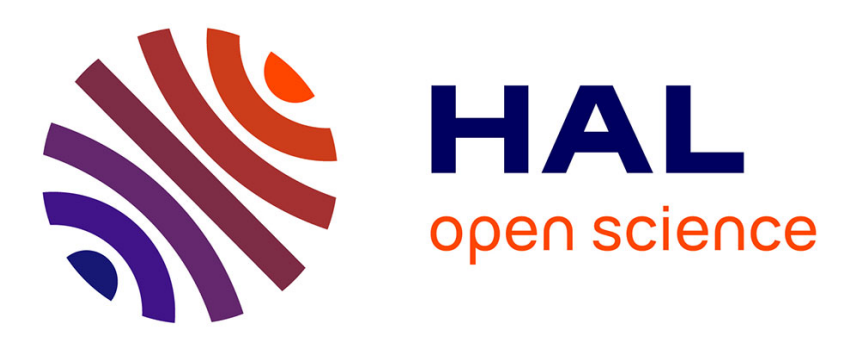

\title{
Correlated blinking of fluorescent emitters mediated by single plasmons
}

D. Bouchet, E. Lhuillier, S. Ithurria, A. Gulinatti, I. Rech, R. Carminati, Y. de Wilde, V. Krachmalnicoff

\section{- To cite this version:}

D. Bouchet, E. Lhuillier, S. Ithurria, A. Gulinatti, I. Rech, et al.. Correlated blinking of fluorescent emitters mediated by single plasmons. Physical Review A: Atomic, molecular, and optical physics [1990-2015], 2017, 95 (3), pp.033828 10.1103/PhysRevA.95.033828 . hal-01509771

\section{HAL Id: hal-01509771 \\ https://hal.science/hal-01509771}

Submitted on 29 May 2017

HAL is a multi-disciplinary open access archive for the deposit and dissemination of scientific research documents, whether they are published or not. The documents may come from teaching and research institutions in France or abroad, or from public or private research centers.
L'archive ouverte pluridisciplinaire HAL, est destinée au dépôt et à la diffusion de documents scientifiques de niveau recherche, publiés ou non, émanant des établissements d'enseignement et de recherche français ou étrangers, des laboratoires publics ou privés. 


\title{
Correlated blinking of fluorescent emitters mediated by single plasmons
}

\author{
D. Bouchet, ${ }^{1}$ E. Lhuillier, ${ }^{2}$ S. Ithurria, ${ }^{3}$ A. Gulinatti, ${ }^{4}$ I. Rech, ${ }^{4}$ R. Carminati, ${ }^{1}$ Y. De Wilde,${ }^{1}$ and V. Krachmalnicoff ${ }^{1, *}$ \\ ${ }^{1}$ ESPCI Paris, PSL Research University, CNRS, Institut Langevin, 1 rue Jussieu, F-75005, Paris, France \\ ${ }^{2}$ Sorbonne Universités, UPMC Univ Paris 06, CNRS-UMR 7588, Institut des NanoSciences de Paris, 4 place Jussieu, 75005 Paris, France \\ ${ }^{3}$ ESPCI Paris, PSL Research University, CNRS, Sorbonne Universités, UPMC University Paris 6; LPEM, 10 rue Vauquelin, \\ F-75231 Paris Cedex 5, France \\ ${ }^{4}$ Politecnico di Milano, Dipartimento di Elettronica, Informazione e Bioingegneria, Piazza da Vinci 32, 20133 Milano, Italy
}

(Received 7 October 2016; published 22 March 2017)

\begin{abstract}
We observe time-correlated emission between a single $\mathrm{CdSe} / \mathrm{CdS} / \mathrm{ZnS}$ quantum dot exhibiting single-photon statistics and a fluorescent nanobead located micrometers apart. This is accomplished by coupling both emitters to a silver nanowire. Single plasmons are created on the latter from the quantum dot, and transfer energy to excite in turn the fluorescent nanobead. We demonstrate that the molecules inside the bead show the same blinking behavior as the quantum dot.
\end{abstract}

DOI: 10.1103/PhysRevA.95.033828

\section{INTRODUCTION}

The study of the correlated emission of two quantum emitters has implications covering a wide panel of fields. It constitutes the keystone for more elaborate experiments crucial for quantum technologies, such as producing entangled states, or realizing interactions among a small ensemble of quantum emitters within the same plasmonic mode along a bus on an integrated device [1], or studying cooperative emission phenomena such as superradiance or subradiance [2]. The communication between two emitters via the exchange of photons propagating in free space was demonstrated so far at temperatures near absolute zero, taking advantage that in that case a molecule with a transition wavelength $\lambda$ resonant with an incoming photon has an extinction cross section on the order of $\lambda^{2} / 2$, which is comparable to the area of a beam focused with a high numerical aperture objective [3]. Here, we address the problem of the communication between two distant nonresonant emitters at room temperature on a nanostructured waveguide. While we use a quantum emitter as a source, we compensate for the low extinction cross section of nonresonant molecules at room temperature using a one-dimensional plasmonic waveguide made of silver and a receiver, which consists of a fluorescent nanobead containing a large amount of fluorescent molecules in near-field coupling with the plasmonic waveguide.

Silver nanowires have been suggested as broadband waveguides, supporting surface plasmons, which propagate over distances of several micrometers along the wire axis [4]. The strong electromagnetic field confinement, which characterizes surface plasmons, leads to large enhancements of the spontaneous emission rate of emitters near-field coupled to silver nanowires. Such enhancement goes along with an efficient coupling into the guided surface plasmon mode, paving the way to applications in quantum nanophotonics [5-7]. It has also been shown that the coupling of single photon emitters, e.g., semiconductor quantum dots (QD) or nitrogen-vacancy defects, to a silver nanowire, generates single surface plasmons exhibiting properties similar to those of single photons

\footnotetext{
*valentina.krachmalnicoff@espci.fr
}

[8,9]. Several theoretical studies highlighted the potential of coupling quantum emitters to surface plasmons [2,10]. Experimental observations of plasmon-assisted energy transfer have so far only involved ensembles of fluorescent emitters [11-13]. In this article, we take advantage of the strong interaction between a single QD and a silver nanowire, as revealed by the measurement of a high Purcell factor $\Gamma / \Gamma_{0} \sim 20\left(\Gamma\right.$ and $\Gamma_{0}$ being the spontaneous decay rates in the presence and in the absence of the nanowire, respectively), and realize long-range energy transfer between this single nanocrystal (donor) and a fluorescent nanobead (acceptor), located on the same nanowire $8.7 \mu \mathrm{m}$ apart. Using time-resolved measurements, we provide a comprehensive analysis of the relation between photon emission from the QD and the fluorescent nanobead.

\section{SAMPLE PREPARATION AND EXPERIMENTAL SETUP}

To prepare the sample, we disperse a dilute solution of chemically synthesized $\mathrm{CdSe} / \mathrm{CdS} / \mathrm{ZnS}$ quantum dots $[14,15]$ (see Appendix E for synthesis details) and polystyrene fluorescent beads (FluoSpheres Microsphere Dark Red, ThermoFisher Scientific, mean diameter $\sim 176 \mathrm{~nm}$ ) on a glass coverslip on which silver nanowires (Sigma Aldrich), with a diameter of $\sim 115 \mathrm{~nm}$ and lengths ranging between 5 and $50 \mu \mathrm{m}$ were previously spin coated. At the end of this procedure, the sample results in isolated silver nanowires near-field coupled with single QDs and single beads. Figure 1(a) presents the optical setup of the experiment, based on an inverted fluorescence microscope combined with timeresolved single photon detection [12].

An oil immersion microscope objective $(\times 100, \mathrm{NA}=1.4)$ located below the sample ensures both illumination and an efficient collection of fluorescence photons. A pulsed laser diode emitting at $\lambda=405 \mathrm{~nm}$ at a repetition rate of $40 \mathrm{MHz}$ is used to excite the QDs, which exhibit large absorption at such wavelength. Acceptor beads have a maximum absorption around $\lambda=640 \mathrm{~nm}$ [see spectra in Fig. 1(b)], and a small absorption at the laser wavelength. Using wide-field images captured with an electron multiplying CCD camera, we select one QD exhibiting single photon statistics (see Appendix A for $g^{(2)}$ photon correlation analysis) and one acceptor bead, both of them located in the near field of a $16 \mu \mathrm{m}$ long silver nanowire. 


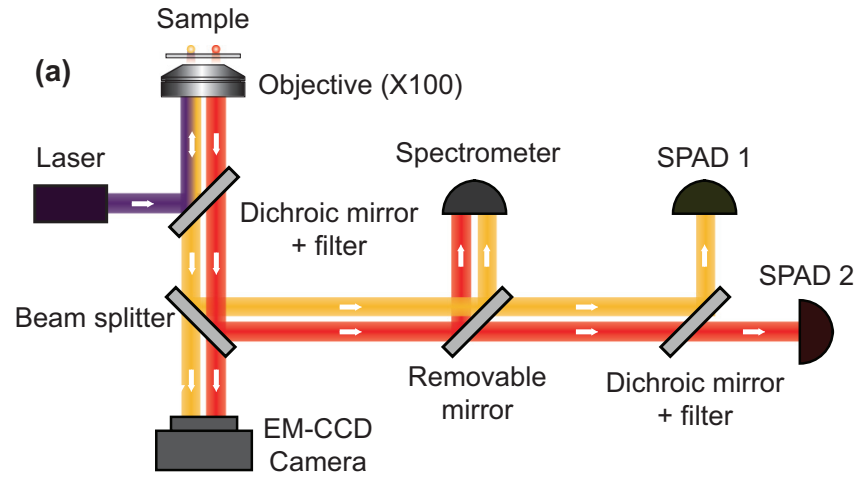

(b)
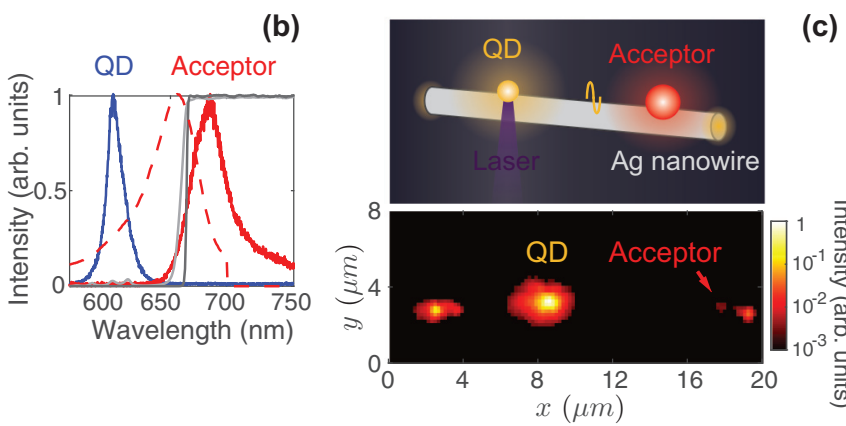

FIG. 1. (a) Optical setup used for imaging, spectroscopy, and time-resolved measurements of a QD (donor) and a fluorescent bead (acceptor) coupled to a silver nanowire. (b) Normalized emission spectra of the QD (in blue) and the acceptor bead (in red). The absorption spectrum of the bead is the red dashed curve. Transmittance of the dichroic mirror and of the long-pass filter are represented in light gray and gray, respectively. (c) Top: Artist view of the experiment. Bottom: Electron multiplying CCD (EM-CCD) taken during the experiment, while the laser is focused on the QD. Bright spots are detected at the position of both emitters as well as both ends of the nanowire.

This configuration is represented in Fig. 1(c) (top). By focusing the laser on the QD, we observe plasmon scattering from the extremities of the nanowire [Fig. 1(c), bottom]. A bright spot also appears at the position of the acceptor. From this image, we determine a distance $d$ of $8.7 \mu \mathrm{m}$ between the QD and the acceptor bead. In the same time, we perform time-resolved measurements using two single photon avalanche diodes (SPADs, PDM-R Micro Photon Devices), using a dichroic mirror and a long-pass filter to split the incident light in such a way that SPAD 1 measures the photon emission from the QD and SPAD 2 measures the emission from the acceptor bead [see Fig. 1(a)]. Fluorescence spectra are measured with a fibered spectrometer (Acton SP2300, Princeton Instruments) and are shown in Fig. 1(b), together with the transmittance of the dichroic mirror and the fluorescence filter. The absence of overlap between the emission spectra and the chosen filters ensures that no photon emitted by the QD is detected on SPAD 2.

\section{EXPERIMENTAL RESULTS}

In order to prove that the acceptor is excited via plasmonmediated energy transfer, we need to characterize the decay (a)
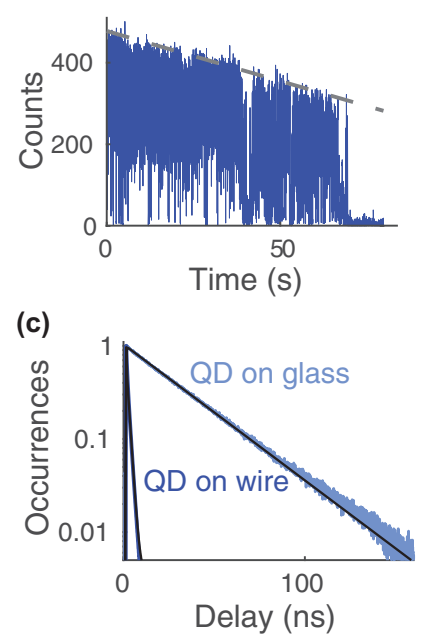

(b)

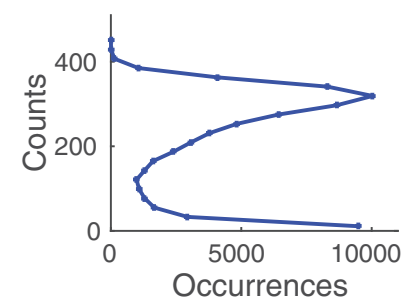

(d)

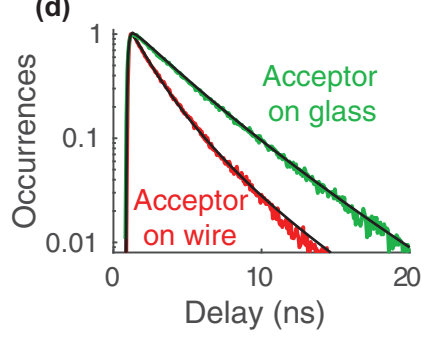

FIG. 2. (a) Time dependence of the fluorescence counts of the $\mathrm{QD}$, time bin equal to $1 \mathrm{~ms}$ (in blue). The drift of the experiment is represented by the dashed line. (b) Distribution of detected events, showing a clear distinction between the bright and the dark state of the QD. (c) Fluorescence decay histograms of a single QD on glass (light blue) and on the nanowire (dark blue). (d) Fluorescence decay histogram for the acceptor on glass and on wire, when excited via a plasmon.

histogram of the QD and the acceptor excited independently. Figure 2(a) presents the time dependence of the fluorescence counts of the QD. Due to the blinking of the QD, we observe strong fluctuations between a bright and a dark state for nearly $70 \mathrm{~s}$ before photobleaching. A slight drift of the experiment from optimum alignment is responsible for a continuous loss of collected signal with time. By applying a linear correction to account for the drift, we retrieve the distribution of detected events [as shown in Fig. 2(b)], where the "on" and the "off" states clearly appear [16]. Besides the antibunching behavior, which we have verified (see Appendix A), this blinking behavior is a signature of the addressing of a single nanocrystal. Fluorescence decay rate $\Gamma$ of the QD, which is the inverse of the excited state lifetime, is measured by fitting the decay histogram with the convolution of the instrument response function (IRF) and a fit function. As expected, $\Gamma$ is greatly enhanced as compared to the decay rate of a single QD on a glass substrate [Fig. 2(c)]. While the decay of a QD on glass presents a monoexponential decay with a decay rate of $0.034 \mathrm{~ns}^{-1}$, the decay statistics of the $\mathrm{QD}$ on the nanowire is biexponential, with $80 \%$ of the total measured photon emission showing a decay rate of $0.67 \mathrm{~ns}^{-1}$. The faster contribution shows a very high decay rate $\left(>12 \mathrm{~ns}^{-1}\right)$ which we attribute to the formation of biexcitons in the QD. From photon coincidence measurements performed on single QDs within the same experimental conditions, we estimate the biexciton-to-exciton ratio to be on the order of $30 \%$ [17], which agrees with the estimate based on the decay histogram.

In order to characterize the acceptor fluorescence, we proceed as follows. Since, in the energy transfer experiment, the acceptor is excited via the plasmon launched by the QD decay, we measure the fluorescence of the acceptor bead when 


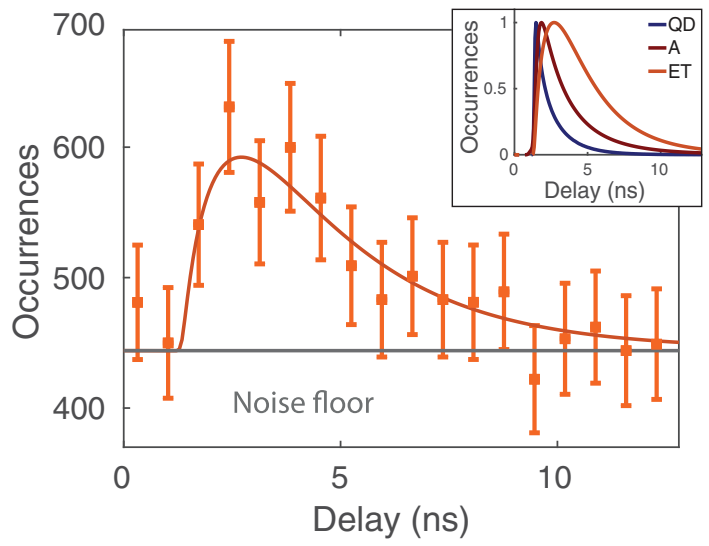

FIG. 3. Measured decay histogram of the acceptor together with the model function (in orange). Error bars correspond to \pm 2 standard deviations (95\% confidence level). Inset: Fit functions of the decay histograms of the QD (blue curve) and the acceptor (A, red curve) excited independently and their convolution (ET, orange curve).

excited by a surface plasmon launched by focusing a pulsed laser at $\lambda=640 \mathrm{~nm}$ onto the left edge of the wire [on the system shown in Fig. 1(c)]. In order to mimic the QD emission, the laser wavelength has been chosen to be close to the QD emission maximum. The decay histogram of the acceptor on glass and on a silver nanowire, while excited via a surface plasmon, is shown in Fig. 2(d). By using a log-normal function to fit the decay histogram and the most frequent values of $\Gamma$ as estimate of the decay rate, we measure a Purcell factor $\Gamma / \Gamma_{0}$ on the order of 2. Interestingly, when the acceptor is coupled to the nanowire, its decay rate is larger when the excitation occurs via the surface plasmon than for a free space excitation (see more experimental details in Appendix B). Indeed, in the first situation, only the molecules inside the bead that are well coupled to the nanowire are excited, while in the second situation excitation concerns all the molecules inside the bead.

The occurrence of energy transfer between the QD and the bead implies that the measured acceptor decay histogram is the convolution of the decay histogram of the donor and acceptor excited independently. The expected signal, for the system studied in Fig. 1(c), is shown in the inset of Fig. 3 (orange curve) and is computed as the convolution of the functions fitting the decay histograms of the donor and the acceptor when excited independently. Experimental data, together with the expected curve, are reported in Fig. 3. Their excellent agreement is a proof that the observed acceptor fluorescence comes from energy transfer from the QD via the surface plasmon. Details on signal processing are reported in Appendix C. From the decay histograms, we estimate the total number of fluorescence photons coming from the acceptor $N_{2}$ to be of $1010 \pm 50$, while we detect at the same time $N_{1}=1.6 \times 10^{7}$ fluorescence photons from the QD.

Since the acceptor is excited via plasmon energy transfer, we expect the QD and the acceptor to blink simultaneously. This can be proved by characterizing the correlation between the fluorescence intensity $I_{1}(t)$ and $I_{2}(t)$ measured for the QD and the acceptor, respectively. Since the QD blinks on ms time scales, we measure the correlation between the photons emitted by the QD and the bead on such temporal delays and we demonstrate simultaneous blinking of the two emitters. Simultaneous blinking will lead to a linear relation, such that $I_{2}(t)=\alpha I_{1}(t)$ with $\alpha=N_{2} / N_{1}$. The degree of correlation, as a function of the delay $\tau$ between the signals detected on each channel, is measured by the coefficient $R(\tau)$ defined as follows:

$$
R(\tau)=\frac{\operatorname{Cov}\left[I_{1}(t), I_{2}(t+\tau)\right]}{\hat{\sigma}_{1} \hat{\sigma}_{2}},
$$

where $\operatorname{Cov}\left[I_{1}(t), I_{2}(t+\tau)\right]$ is the covariance of the two intensities and $\hat{\sigma}_{1}$ and $\hat{\sigma}_{2}$ are estimates of the standard deviation for the fluorescence intensities $I_{1}$ and $I_{2}$, respectively. With this definition, it follows from the Cauchy-Schwartz inequality that $R$ is equal to 1 for perfectly correlated variables while it vanishes for uncorrelated variables. In our experiment, the major contribution to the variance of the QD fluorescence is blinking, which dominates on noise. Therefore, we can use the standard deviation $\sigma_{1}$ measured in channel 1 to approximate $\hat{\sigma_{1}} \approx \sigma_{1}$ and, since fluorescence of the QD and of the acceptor are linearly dependent, $\hat{\sigma}_{2} \approx \alpha \sigma_{1}$ (see Appendix D for the mathematical derivation). We calculate the covariance coefficient $R(\tau)$ of the centered values of the intensity traces measured on SPAD 1 and SPAD 2, with a time resolution of $1 \mathrm{~ms}$. In order to have a large variance in the signals, we select a $30 \mathrm{~s}$ time interval [from $t=40-70 \mathrm{~s}$ in Fig. 2(a)] during which the QD blinks frequently. We symmetrize the intensity time traces to be insensitive to any drift of the experiment. Furthermore, in order to improve the signal to noise ratio, we select the photons on the basis of their arrival time with respect to the laser excitation pulse. For channel 2 (acceptor fluorescence), this amounts to consider the interval between $1.856 \mathrm{~ns}$ and $12 \mathrm{~ns}$.

We show the correlation coefficient $R(\tau)$ of the QD and the acceptor intensity traces in Fig. 4(a) (orange curve). We observe that, while $R$ goes to zero for large delays, the degree of linear correlation between the two intensity traces is almost one for zero delay, proving that the QD (a)
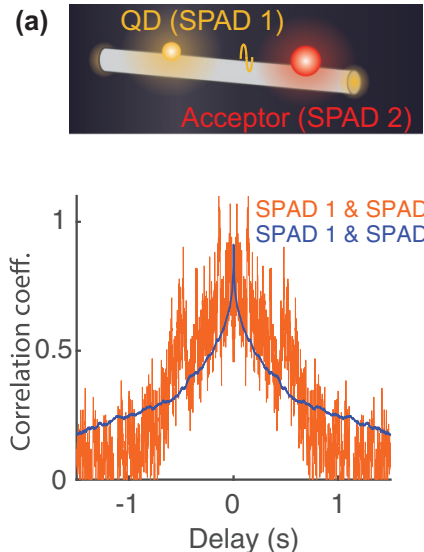

(b)

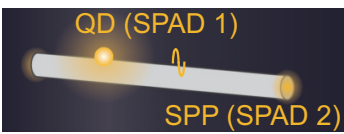

FIG. 4. (a) Correlation coefficient of the QD fluorescence (SPAD 1) and the acceptor fluorescence (SPAD 2) as defined in Eq. (1). (b) Correlation coefficient for an experiment in which we measure the QD fluorescence (SPAD 1) and the light scattered by the nanowire end (SPAD 2). 
and the acceptor blink simultaneously. The small deviation from unity is a consequence of the approximation $\hat{\sigma}_{1} \approx \sigma_{1}$. In the same figure, we show the correlation coefficient $R(\tau)$ of the intensity trace of the QD with itself (blue curve). The similarity between the two curves, without any normalization, proves that the time scales of the fluctuations are effectively the same for both the QD and the acceptor fluorescence. For comparison, we performed an experiment in which a single QD launches a plasmon on a silver nanowire and we detected the light scattered at the nanowire end, located $7 \mu \mathrm{m}$ apart. Figure 3(b) shows the correlation coefficient of the QD fluorescence intensity and the intensity of the scattered at the wire extremity (black curve), as well as the autocorrelation of the QD trace (blue curve). The similarity between the two curves corroborates our analysis.

\section{CONCLUSION}

Our work shows that it is possible to excite a fluorescent molecule embedded in a nanobead by means of single plasmons launched by a single quantum emitter. Indeed, photon emission from the QD and the nanobead are linearly related, which implies that the nanobead exhibits the same blinking behavior of the QD. Note that an anticorrelated emission between the QD and the acceptor is expected on the ns time scale but its observation is hindered by the noise level due to the weak efficiency of the energy transfer process. A significant enhancement of the energy transfer efficiency would be achieved using structures with optimized mode coupling between the donor and the acceptor sites. In this perspective, hybrid plasmon-dielectric waveguides appear as promising structures that would allow an enhancement of the energy transfer efficiency of several orders of magnitude [18,19]. The use of techniques of deterministic nanopositioning of quantum emitters will allow us to further optimize the coupling. Ultimately, efficient coupling of single emitters via plasmonic modes opens new avenues to investigate cooperative emission phenomena such as superradiance, which could be probed by measuring the dependence of the decay rate of an ensemble of similar emitters along a metallic nanowire as a function of their number.

\section{ACKNOWLEDGMENTS}

The authors thank N. Lequeux and T. Pons for helping in sample preparation. This work was supported by LABEX WIFI (Laboratory of Excellence ANR-10-LABX-24) within the French Program Investments for the Future under reference ANR-10- IDEX-0001-02 PSL*, by the Region Ile-de-France in the framework of DIM Nano-K, by the Programme Emergences 2015 of the City of Paris, by PSL Research University in the framework of the project COSINE and by ANR Nanodose.

\section{APPENDIX A: PHOTON COINCIDENCE MEASUREMENTS OF SINGLE QUANTUM DOTS}

In this section we present experimental data of photon coincidence measurements performed on single $\mathrm{CdSe} / \mathrm{CdS} / \mathrm{ZnS}$ quantum dots. For a single nanocrystal on a glass coverslip
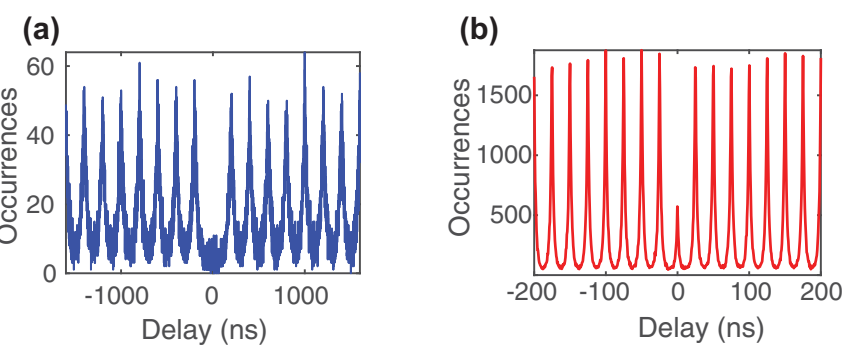

FIG. 5. (a) Single CdSe/CdS/ZnS nanocrystal on glass. Repetition rate: $5 \mathrm{MHz}$. Measured fluorescent counts of the bright state: $20 \mathrm{c} / \mathrm{ms}$. (b) Single CdSe/CdS/ZnS nanocrystal on silver nanowire. Repetition rate: $40 \mathrm{MHz}$. Measured fluorescent counts of the bright state: $300 \mathrm{c} / \mathrm{ms}$.

excited with an average laser power of $\sim 0.5 \mu \mathrm{W}$ (repetition rate $5 \mathrm{MHz}$, pulse duration $600 \mathrm{ps}$ ) we measure a strong antibunching [see Fig. 5(a)]. When increasing the excitation power to the one used to perform the measurements reported in the manuscript (average power of $\sim 2 \mu \mathrm{W}$, repetition rate $40 \mathrm{MHz}$, pulse duration $600 \mathrm{ps}$ ), the second-order correlation function shows a small peak at zero delay, characteristic of the formation of biexcitons see Fig. 5(b). By measuring the ratio between the area under the zero delay peak and the adjacent ones, we find a biexciton-to-exciton ratio of $4 \%$ and $29 \%$, respectively.

\section{APPENDIX B: DECAY RATE OF THE ACCEPTOR BEAD COUPLED TO A SILVER NANOWIRE}

In this section we present the measurement of the decay rate of the acceptor bead when the excitation does not occur via energy transfer. The different excitations performed are schematically reported in Fig. 6(a) and the corresponding decay histograms are reported in Fig. 6(b) following the same color scheme as in Fig. 6(a). As a reference, we measured the decay histogram of the acceptor on a glass coverslip (blue curve). Then we compared it to the decay histogram obtained for the acceptor bead coupled to a silver nanowire in two different situations. The bead can either be excited by a laser ( $\lambda=642 \mathrm{~nm}$ ) focused on it (green curve) or by a surface plasmon propagating on the silver wire (red curve). The plasmon is launched by focusing a laser $(\lambda=642 \mathrm{~nm})$ on the extremity of the wire.

As expected, the decay rate of the bead coupled to the wire (for both excitation schemes) is larger than on glass. Moreover, the decay rate obtained when the bead is excited via the surface plasmon is larger than the one obtained with a far-field excitation. Indeed, in the first situation, only the acceptor molecules located closer to the wire are excited, while in the second situation all the molecules in the bead are excited. In order to get a quantitative measurement of the decay rate, we fit the decay histograms with the convolution of the instrument response function (IRF) and a log-normal distribution of decay rates which reads:

$$
I_{\log }(t)=A \int_{\Gamma=0}^{\infty} \Phi(\Gamma) \exp (-\Gamma t) d \Gamma
$$


(a)
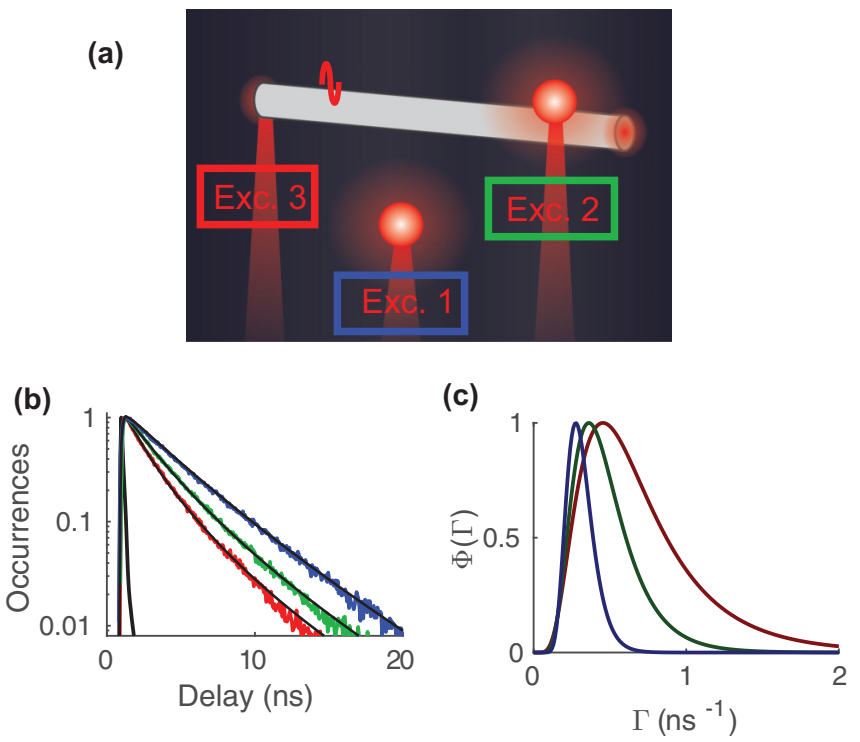

FIG. 6. (a) Scheme of the experiment. (b) Measured decay histogram in three different situations: bead not coupled to a nanowire (blue), bead coupled to a nanowire and excited directly by the laser (green), same bead excited by a surface plasmon launched by focusing a laser on the extremity of the nanowire (red). (c) Distribution of decay rates for these three situations.

with

$$
\Phi(\Gamma)=\exp \left(-\frac{\ln ^{2}\left(\Gamma / \Gamma_{m f}\right)}{w^{2}}\right) .
$$

In the formula above, $A$ is the amplitude, $\Gamma_{m f}$ the most frequent decay rate, and $w$ a parameter characterizing the width of the distribution. Figure 6(c) shows the measured distribution of decay rates $\Phi(\Gamma)$ for the three situations depicted in Fig. 6(a).

\section{APPENDIX C: ENERGY TRANSFER DECAY HISTOGRAM ANALYSIS}

In this section we present raw acceptor decay histogram and the data processing that has been made on it in order to prove the occurrence of the energy transfer. Figure 7(a) presents raw data, measured with a 64 ps resolution. The decay histogram shows two components. The first one is characterized by a long lifetime, characteristic of the occurrence of energy transfer. The second one is characterized by a very short lifetime, which is well fitted by the instrument response function of the experi-
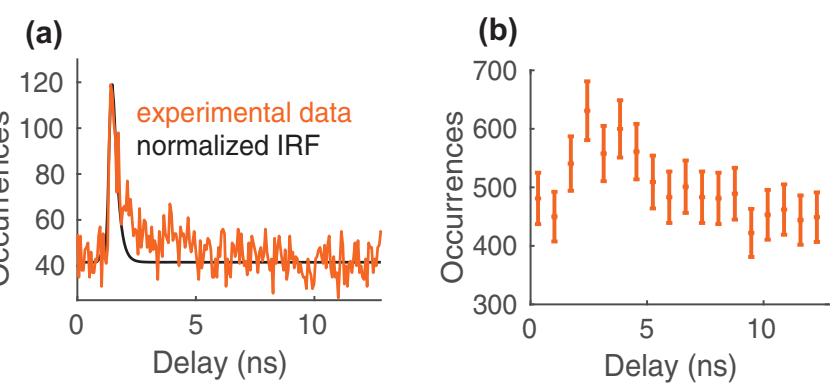

FIG. 7. (a) Experimental data binned at 64 ps (in orange). The black curve is a fit of the data by the IRF. (b) Corrected data binned at $700 \mathrm{ps}$. mental setup. Such fast component of the decay histogram has been observed in a test experiment, performed on bare silver nanowires deposited on a glass coverslip under pulsed excitation at $\lambda=405 \mathrm{~nm}$ and is attributed to silver luminescence. Luminescence is characterized by a very broad spectrum and can propagate along the nanowire via a surface plasmon, finally being scattered by the acceptor bead and therefore detected.

We removed this contribution by fitting the instrument response function to the data and by subtracting it from the decay histogram. Figure 7(b) shows the corrected decay histogram with a $700 \mathrm{ps}$ resolution. As explained in the main text, error bars correspond to \pm 2 standard deviations $(95 \%$ confidence level).

\section{APPENDIX D: CHARACTERIZATION OF THE DEGREE OF LINEAR CORRELATION BETWEEN INTENSITY TIME TRACES}

In this section we characterise the degree of linear correlation between two intensity time traces $I_{1}(t)$ and $I_{2}(t)$. Each time trace $i$ can be written as a sum of the fluorescence counts $I_{i}^{\prime}(t)$ and the noise counts $I_{i}^{\prime \prime}(t)$, reading

$$
\begin{aligned}
& I_{1}(t)=I_{1}^{\prime}(t)+I_{1}^{\prime \prime}(t) \\
& I_{2}(t)=I_{2}^{\prime}(t)+I_{2}^{\prime \prime}(t) .
\end{aligned}
$$

We define $\overline{I_{1}}$ and $\overline{I_{2}}$ as the mean value on the time interval $\Delta t$ of, respectively, $I_{1}(t)$ and $I_{2}(t)$. Similarly, we write

$$
\begin{aligned}
& \overline{I_{1}}=\overline{I_{1}^{\prime}}+\overline{I_{1}^{\prime \prime}} \\
& \overline{I_{2}}=\overline{I_{2}^{\prime}}+\overline{I_{2}^{\prime \prime}} .
\end{aligned}
$$

On this time interval $\Delta t$, the covariance of the intensity time traces reads

$$
\operatorname{Cov}\left[I_{1}(t), I_{2}(t)\right]=\int_{\Delta t}\left[I_{1}(t)-\overline{I_{1}}\right]\left[I_{2}(t)-\overline{I_{2}}\right] d t .
$$

It follows

$$
\begin{aligned}
\operatorname{Cov}\left[I_{1}(t), I_{2}(t)\right]= & \int_{\Delta t}\left[I_{1}^{\prime}(t)-\overline{I_{1}^{\prime}}\left[\left[I_{2}^{\prime}(t)-\overline{I_{2}^{\prime}}\right] d t\right.\right. \\
& +\int_{\Delta t}\left[I_{1}^{\prime}(t)-\overline{I_{1}^{\prime}}\right]\left[I_{2}^{\prime \prime}(t)-\overline{I_{2}^{\prime \prime}}\right] d t \\
& +\int_{\Delta t}\left[I_{1}^{\prime \prime}(t)-\overline{I_{1}^{\prime \prime}}\right]\left[I_{2}^{\prime}(t)-\overline{I_{2}^{\prime}}\right] d t \\
& +\int_{\Delta t}\left[I_{1}^{\prime \prime}(t)-\overline{I_{1}^{\prime \prime}}\right]\left[I_{2}^{\prime \prime}(t)-\overline{I_{2}^{\prime \prime}}\right] d t .
\end{aligned}
$$

The last three contributions involve the integral over $\Delta t$ of a white noise and thus cancel out. The covariance is therefore determined by the fluorescence counts only, and reads

$$
\operatorname{Cov}\left[I_{1}(t), I_{2}(t)\right]=\int_{\Delta t}\left[I_{1}^{\prime}(t)-\overline{I_{1}^{\prime}}\right]\left[I_{2}^{\prime}(t)-\overline{I_{2}^{\prime}}\right] d t .
$$

This equation equals zero if $I_{1}^{\prime}(t)$ and $I_{2}^{\prime}(t)$ are uncorrelated. However, if a linear relation between $I_{1}^{\prime}(t)$ and $I_{2}^{\prime}(t)$ exists such that $I_{2}^{\prime}(t)=\alpha I_{1}^{\prime}(t)$, this equation reads

$$
\operatorname{Cov}\left[I_{1}(t), I_{2}(t)\right]=\alpha \times \int_{\Delta t}\left[I_{1}^{\prime}(t)-\overline{I_{1}^{\prime}}\right]^{2} d t .
$$


We define the correlation coefficient $R$ as follows:

$$
R=\frac{\operatorname{Cov}\left[I_{1}(t), I_{2}(t)\right]}{\alpha \times \int_{\Delta t}\left[I_{1}^{\prime}(t)-\overline{I_{1}^{\prime}}\right]^{2} d t} .
$$

We define $\sigma_{1}^{\prime}$ and $\sigma_{2}^{\prime}$ as the standard deviation on the time interval $\Delta t$ of the fluorescence contribution of each trace, namely, $I_{1}^{\prime}(t)$ and $I_{2}^{\prime}(t)$. They read:

$$
\begin{aligned}
& \sigma_{1}^{\prime}=\sqrt{\int_{\Delta t}\left[I_{1}^{\prime}(t)-\overline{I_{1}^{\prime}}\right]^{2} d t} \\
& \sigma_{2}^{\prime}=\sqrt{\int_{\Delta t}\left[I_{2}^{\prime}(t)-\overline{I_{2}^{\prime}}\right]^{2} d t .}
\end{aligned}
$$

From $I_{2}^{\prime}(t)=\alpha I_{1}^{\prime}(t)$, we can directly write $\sigma_{1}^{\prime}=\alpha \sigma_{2}^{\prime}$. With these notations, the correlation coefficient $R$ reads

$$
R=\frac{\operatorname{Cov}\left[I_{1}(t), I_{2}(t)\right]}{\sigma_{1}^{\prime} \sigma_{2}^{\prime}} .
$$

Using this definition,

(i) if $I_{1}^{\prime}(t)$ and $I_{2}^{\prime}(t)$ are uncorrelated then $R=0$,

(ii) if $I_{1}^{\prime}(t)$ and $I_{2}^{\prime}(t)$ are linearly related then $R=1$.

\section{APPENDIX E: SYNTHESIS OF CdS/CdSe/ZnS NANOCRYSTALS}

\section{Chemicals}

1-Octadecene (ODE, 90\%, Aldrich), oleylamine (70\%, Fluka), oleic acid (90\%, Aldrich), sodium myristate $(99 \%$, Fluka), cadmium nitrate (99.999\%, Aldrich), cadmium oxide (99.99\%, Aldrich), zinc nitrate (aldrich, 98\%), sulfur powder (Aldrich, 99;998\%), selenium powder 100 mesh (99.99\%, Aldrich), sulfur (99.998\%, Aldrich), ethanol (Carlo Erba, 99.5\%), methanol (VWR, 100\%), n methyl formamide (NMF) (Aldrich, 99\%).

\section{Precursor preparation}

$\mathrm{Cd}(\mathrm{Myr})_{2}: 3.2 \mathrm{~g}(80 \mathrm{mmol})$ of $\mathrm{NaOH}$ are dissolved in $500 \mathrm{~mL}$ of methanol. Then $18.2 \mathrm{~g}$ of myristic acid are added in the flask. The whitish solution is stirred for $15 \mathrm{~min}$. Meanwhile, $8.2 \mathrm{~g}$ of cadmium nitrate tetrahydrate are dissolved in $50 \mathrm{~mL}$ of methanol. This solution is added to the sodium myristate solution and a white precipitate gets formed. The solution is further stirred for $15 \mathrm{~min}$. The white solid is isolated by filtration and washed several times with methanol. The solid is finally dried under vacuum overnight.

S-ODE 0.1M: $150 \mathrm{~mL}$ of octadecene are degassed under vacuum for $30 \mathrm{~min}$. Then the flask is put under $\mathrm{Ar}$ and $480 \mathrm{mg}$ of $\mathrm{S}$ powder are introduced in the flask. The solution is heated at $140^{\circ} \mathrm{C}$ until the formation of a yellow clear solution. The flask is finally cooled down.
Se-ODE 0.1 M: $140 \mathrm{~mL}$ of octadecene are degassed under vacuum for $30 \mathrm{~min}$. Then the flask is put under $\mathrm{Ar}$ and the temperature raised to $170^{\circ} \mathrm{C} .1 .18 \mathrm{~g}$ of Se powder is mixed in $10 \mathrm{~mL}$ of octadecene and introduced in the flask dropwise. The temperature is finally raised to $205^{\circ} \mathrm{C}$ and the flask heated for $30 \mathrm{~min}$. The final solution is clear and yellow-orange. The flask is finally cooled down.

$\mathrm{Cd}(\mathrm{OA})_{2} 0.5 \mathrm{M}$ : In a $250 \mathrm{ml}$ three-neck flask, $6.42 \mathrm{~g}$ of CdO and $100 \mathrm{~mL}$ of oleic acid are introduced and the mixture is heated at $180^{\circ} \mathrm{C}$ until the formation of a yellow clear solution. Then the flask is cooled below $120^{\circ} \mathrm{C}$ and put under vacuum for $30 \mathrm{~min}$. The flask is finally cooled down.

\section{QD synthesis}

In a three-neck flask we introduce $170 \mathrm{mg}$ of cadmium myristate with $7.5 \mathrm{~mL}$ of octadecene. The flask is degassed under vacuum at room temperature for $30 \mathrm{~min}$. The atmosphere is then switched to $\mathrm{Ar}$ and the temperature raised to $250^{\circ} \mathrm{C}$. $12 \mathrm{mg}$ of selenium powder are mixed with $1 \mathrm{~mL}$ of ODE and sonicated, before being quickly injected into the flask. After $5 \mathrm{~min}$ at $250^{\circ} \mathrm{C}, 0.2 \mathrm{~mL}$ of oleic acid and $2 \mathrm{~mL}$ of oleylamine are added. Then a mixture of $1 \mathrm{~mL}$ cadmium oleate $(0.5 \mathrm{M})$ and $5 \mathrm{~mL}$ of Se-ODE $(0.1 \mathrm{M})$ is prepared and half of it is injected with a $5 \mathrm{~mL} / \mathrm{h}$ flow rate. The reaction is then cooled down to room temperature and the nanocrystal precipitated by addition of ethanol. After centrifugation the QD pellet is redispersed in $10 \mathrm{~mL}$ of octadecene.

For the growth of the CdS shell, A SILAR method is used, where we successively introduce precursor for Cd and S. $4 \mathrm{~mL}$ of the previous solution of CdSe core are mixed with $16 \mathrm{~mL}$ of octadecene and $10 \mathrm{~mL}$ of oleylamine. The solution is degassed for $30 \mathrm{~min}$. Then cadmium oleate $(0.1 \mathrm{M})$ and S-ODE $(0.1 \mathrm{M})$ are introduced in the flask and the temperature raised to $230^{\circ} \mathrm{C}$. After $20 \mathrm{~min}$, cadmium oleate $(0.1 \mathrm{M})$ is added and after $10 \mathrm{~min}$ S-ODE $(0.1 \mathrm{M})$ is added. We repeat this addition of $\mathrm{Cd}$ and $\mathrm{S}$ up to the formation of four layers of $\mathrm{CdS}$. The reaction is then cooled down to room temperature and the nanocrystal precipitated by addition of ethanol. After centrifugation the QD pellet is redispersed in $10 \mathrm{~mL}$ of octadecene.

For the growth of $\mathrm{ZnS}$ shell use the c-ALD procedure as developed in Ref. [15]. Briefly, a $0.2 \mathrm{M}$ solution of $\mathrm{Na}_{2} \mathrm{~S}$ in $\mathrm{N}$ methyl formamide (NMF) and a $0.2 \mathrm{M}$ solution of $\mathrm{Zn}\left(\mathrm{NO}_{3}\right)_{2} \cdot 6 \mathrm{H}_{2} \mathrm{O}$ in NMF are prepared. The CdSe/CdS QD dispersed in hexane are mixed with the same amount of NMF. Some of the $\mathrm{Na}_{2} \mathrm{~S}$ solution is added and the solution is stirred until we observe a phase transfer. The particles are then precipitated by addition of ethanol and centrifuge. The formed pellet is redispersed in fresh NMF. Some of the zinc solution is added and the same cleaning procedure is repeated. The growth of a second $\mathrm{ZnS}$ layer is conducted. The particles are precipitated and cleaned again and an excess of oleic acid is added to the vial to retransfer the QD toward a nonpolar phase.
[1] S. Faez, P. Türschmann, H. R. Haakh, S. Götzinger, and V. Sandoghdar, Coherent Interaction of Light and Single Molecules in a Dielectric Nanoguide, Phys. Rev. Lett. 113, 213601 (2014).
[2] D. Martin-Cano, L. Martin-Moreno, F. J. Garcia-Vidal, and E. Moreno, Resonance Energy transfer and superradiance mediated by plasmonic nanowaveguides, Nano Lett. 10, 3129 (2010). 
[3] Y. L. A. Rezus, S. G. Walt, R. Lettow, A. Renn, G. Zumofen, S. Götzinger, and V. Sandoghdar, Single-Photon Spectroscopy of a Single Molecule, Phys. Rev. Lett. 108, 093601 (2012).

[4] H. Ditlbacher, A. Hohenau, D. Wagner, U. Kreibig, M. Rogers, F. Hofer, F. R. Aussenegg, and J. R. Krenn, Silver Nanowires as Surface Plasmon Resonators, Phys. Rev. Lett. 95, 257403 (2005).

[5] D. E. Chang, A. S. Sørensen, P. R. Hemmer, and M. D. Lukin, Quantum Optics with Surface Plasmons, Phys. Rev. Lett. 97, 053002 (2006).

[6] D. E. Chang, A. S. Sørensen, E. A. Demler, and M. D. Lukin, A single-photon transistor using nanoscale surface plasmons, Nature Phys. 3, 807 (2007).

[7] M. S. Tame, K. R. McEnery, Ş. K. Özdemir, J. Lee, S. A. Maier, and M. S. Kim, Quantum plasmonics, Nature Phys. 9, 329 (2013).

[8] A. V. Akimov et al., Generation of single optical plasmons in metallic nanowires coupled to quantum dots, Nature (London) 450, 402 (2007).

[9] R. Kolesov et al., Waveparticle duality of single surface plasmon polaritons, Nature Phys. 5, 470 (2009).

[10] D. Dzsotjan, A. S. Sørensen, and M. Fleischhauer, Quantum emitters coupled to surface plasmons of a nanowire: A Green's function approach, Phys. Rev. B 82, 075427 (2010).

[11] P. Andrew and W. L. Barnes, Energy transfer across a metal film mediated by surface plasmon polaritons, Science 306, 1002 (2004).
[12] D. Bouchet, D. Cao, R. Carminati, Y. De Wilde, and V. Krachmalnicoff, Long-Range Plasmon-Assisted Energy Transfer between Fluorescent Emitters, Phys. Rev. Lett. 116, 037401 (2016).

[13] J. de Torres, P. Ferrand, G. Colas des Francs, and J. Wenger, Coupling emitters and silver nanowires to achieve long-range plasmon-mediated fluorescence energy transfer, ACS Nano 10, 3968 (2016).

[14] D. V. Talapin et al., CdSe/CdS/ZnS and CdSe/ZnSe/ZnS Core-Shell-Shell nanocrystals, J. Phys. Chem. B 108, 18826 (2004).

[15] S. Ithurria and D. V. Talapin, Colloidal atomic layer deposition (c-ALD) using self-limiting reactions at nanocrystal surface coupled to phase transfer between polar and nonpolar media, J. Am. Chem. Soc. 134, 18585 (2012).

[16] B. Mahler et al., Towards non-blinking colloidal quantum dots, Nature Mater. 7, 659 (2008).

[17] G. Nair, J. Zhao and M. G. Bawendi, Biexciton quantum yield of single semiconductor nanocrystals from photon statistics, Nano Lett. 11, 1136 (2011).

[18] R. F. Oulton, V. J. Sorger, D. A. Genov, D. F. P. Pile, and $\mathrm{X}$. Zhang, A hybrid plasmonic waveguide for subwavelength confinement and long-range propagation, Nature Photon. 2, 496 (2008)

[19] P. M. d. Roque, N. F. v. Hulst, and R. Sapienza, Nanophotonic boost of intermolecular energy transfer, New J. Phys. 17, 113052 (2015). 